\title{
AN ASYMPTOTIC FUGLEDE THEOREM
}

\author{
ROBERT MOORE
}

ABSTRACT. The main result is that if an operator $B$ on Hilbert space "almost" commutes with a normal operator $N$, then $B$ almost commutes with $N^{*}$ as well. The Theorem is then extended to a Putnam-like version which states that if $B$ almost intertwines two normal operators, then it almost intertwines continuous functions of those operators.

Let $N$ be a normal operator on Hilbert space with spectral measure $E$, and let $B$ be any bounded operator. Two common equivalent formulations of the Fuglede theorem (for example, see [3]) are the following:

(i) If $N B=B N$, then $N^{*} B=B N^{*}$.

(ii) If $N B=B N$, then $E(S) B=B E(S)$ for all Borel sets $S$.

Is an approximate version of the se statements true? That is, if $\|N B-B N\|$ is "small," is $\left\|N^{*} B-B N^{*}\right\|$ small? Is $\|E(S) B-B E(S)\|$ small? Bastian and Harrison [1] showed that the answer to the second question is "no," by constructing a normal operator $N$, a Borel set $S$, and a sequence $\left\{B_{n}\right\}$ for which $\left\|B_{n}\right\|=1$ for all $n$ and $\left\|N B_{n}-B_{n} N\right\| \rightarrow 0$, but $\left\|E(S) B_{n}-B_{n} E(S)\right\|=1$ for all $n$. Johnson and Williams [4] have shown the existence of a normal operator $N$ and a sequence $\left\{B_{n}\right\}$ such that $\left\|B_{n} N-N B_{n}\right\| \rightarrow 0$ but $\left\|N^{*} B_{n}-B_{n} N^{*}\right\| \geq 1$ for all $n$. Hence the answer to the first question is also negative.

In the Johnson-Williams example, the operators $\left\{B_{n}\right\}$ are not uniformly bounded. The purpose of this note is to show that in case $\|B\|$ is under control, then the answer to the first question is affirmative. Our techniques were inspired by those of Rosenblum [5].

Theorem. Let $N$ be a normal operator. If $K$ and $\epsilon$ are greater than 0 , then there is $\delta$ greater than 0 such that $\|B\| \leq K$ and $\|N B-B N\| \leq \delta$ imply $\left\|N^{*} B-B N^{*}\right\| \leq \epsilon$.

Proof. Without loss of generality we assume that $\|N\| \leq 1$, and that $K=1$. Let $B$ be an operator with $\|B\| \leq 1$, and set $\|N B-B N\|=\eta$. A

Received by the editors April 17, 1974.

AMS (MOS) subject classifications (1970). Primary 47B 15.

Key words and phrases. Normal operator, Fuglede theorem, spectral measure. 
well-known inductive argument shows that

$$
\left\|N^{k} B-B N^{k}\right\| \leq k\|N\|^{k-1}\|N B-B N\| \leq k \eta,
$$

for positive integers $k$. Thus, for any complex number $\lambda$,

$$
\begin{aligned}
\left\|e^{i \bar{\lambda} N} B-B e^{i \bar{\lambda} N}\right\| & =\left\|\sum_{k=0}^{\infty} \frac{(i \bar{\lambda})^{k}}{k !}\left(N^{k} B-B N^{k}\right)\right\| \\
& \leq \sum_{k=0}^{\infty} \frac{|\lambda|^{k}}{k !}\left\|N^{k} B-B N^{k}\right\| \leq \sum_{k=0}^{\infty} \frac{|\lambda|^{k}}{k !} k \eta \\
& =\eta|\lambda| \sum_{k=1}^{\infty} \frac{|\lambda|^{k-1}}{(k-1) !}=\eta|\lambda| e^{|\lambda|} .
\end{aligned}
$$

Similarly we can show that $e^{|\lambda|}$ bounds each of the norms $\left\|e^{i \bar{\lambda} N}\right\|$, $\left\|e^{-i \lambda N^{*}}\right\|$, and $\left\|e^{i \lambda N^{*}}\right\|$. Hence

$$
\left\|e^{i \lambda N^{*}}\left(e^{i \bar{\lambda} N} B-B e^{i \bar{\lambda} N}\right) e^{-i \bar{\lambda} N} e^{-i \lambda N^{*}}\right\| \leq \eta|\lambda| e^{4|\lambda|} .
$$

Since $N$ is norinal we can add exponents to get

$$
\left\|e^{i\left(\bar{\lambda} N+\lambda N^{*}\right)} B e^{-i\left(\bar{\lambda} N+\lambda N^{*}\right)}-e^{i \lambda N^{*}} B e^{-i \lambda N^{*}}\right\| \leq \eta|\lambda| e^{4|\lambda|}
$$

or

$$
\left\|e^{i \lambda N^{*}} B e^{-i \lambda N^{*}}\right\| \leq\left\|e^{i\left(\bar{\lambda} N+\lambda N^{*}\right)} B e^{-i\left(\bar{\lambda} N+\lambda N^{*}\right)}\right\|+\eta|\lambda| e^{4|\lambda|} .
$$

But $\bar{\lambda} N+\lambda N^{*}$ is Hermitian, so $e^{i\left(\bar{\lambda} N+\lambda N^{*}\right)}$ is unitary, and the first term on the right-hand side of the last.inequality is equal to $\|B\|$ for all values of $\lambda$. Thus

$$
\left\|e^{i \lambda N^{*}} B e^{-i \lambda N^{*}}\right\| \leq\|B\|+\eta|\lambda| e^{4|\lambda|} \leq 1+\eta|\lambda| e^{4|\lambda|} .
$$

The function $\phi$ defined by $\phi(\lambda)=e^{i \lambda N^{*}} B e^{-i \lambda N^{*}}$ is an analytic operator-valued function of $\lambda$. By Cauchy's integral formula we have

$$
\phi^{\prime}(0)=\frac{1}{2 \pi i} \int_{\Gamma} \frac{\phi(\lambda)}{\lambda^{2}} d \lambda
$$

where $\Gamma$ is any closed Jordan curve whose interior contains the origin. In case $\Gamma$ is a circle of radius $r$ with center at the origin we get the estimate

$$
\left\|\phi^{\prime}(0)\right\| \leq \frac{2 \pi r}{2 \pi} \cdot \frac{1}{r^{2}} \cdot \max _{\Gamma}\|\phi(\lambda)\| \leq \frac{1}{r}\left(1+\eta r e^{4 r}\right)=\frac{1}{r}+\eta e^{4 r} \text {. }
$$


Notice that

$$
\phi^{\prime}(\lambda)=i N^{*} e^{i \lambda N^{*}} B e^{-i \backslash N^{*}}-i e^{i \lambda N^{*}} B N^{*} e^{-i \lambda N^{*}},
$$

so that $\left\|\phi^{\prime}(0)\right\|=\left\|N^{*} B-B N^{*}\right\|$. Hence

$$
\left\|N^{*} B-B N^{*}\right\| \leq 1 / r+\eta e^{4 r},
$$

and this equation holds for all $r>0$. Thus if $\epsilon>0$, choose $r=2 / \epsilon$ and choose $\delta=1 / 2 \epsilon e^{-4 r}$. Then when $\|N B-B N\| \leq \delta$, that is, $\eta \leq \delta$, we have

$$
\left\|N^{*} B-B N^{*}\right\| \leq 1 / 2 \epsilon+1 / 2 \epsilon e^{-4 r} e^{4 r}=\epsilon \text {. }
$$

This completes the proof.

S. K. Berberian's (by now well-known) trick [2] allows us to extend the result to a Putnam-like version:

Corollary 1. Let $N_{1}$ and $N_{2}$ be normal. If $K$ and $\epsilon$ are greater than zero, there exists $\delta$ greater than zero such that $\|B\| \leq K$ and $\left\|N_{1} B-B N_{2}\right\|$ $\leq \delta$ imply $\left\|N_{1}^{*} B-B N_{2}^{*}\right\| \leq \epsilon$.

Proof. Let $\mathcal{H}$ be the 'Ailbert space on which $N_{1}, N_{2}$, and $B$ act. Define operators $\tilde{N}$ and $\tilde{B}$ on the space $\mathcal{H} \oplus \mathcal{H}$ by

$$
\widetilde{N}=\left(\begin{array}{cc}
N_{2} & 0 \\
0 & N_{1}
\end{array}\right), \quad \widetilde{B}=\left(\begin{array}{ll}
0 & 0 \\
B & 0
\end{array}\right) \text {. }
$$

Then $\|\tilde{B}\|=\|B\|,\left\|N_{1} B-B N_{2}\right\|=\|\tilde{N B}-\tilde{B N}\|$, and $\left\|N_{1}^{*} B-B N_{2}^{*}\right\|=$ $\left\|\tilde{N}^{*} \tilde{B}-\widetilde{B N}^{*}\right\|$. Application of the Theorem to $\tilde{N}$ and $\widetilde{B}$ gives the corollary.

Corollary 2. Let $N_{1}$ and $N_{2}$ be normal and let $\psi$ be a complex-valued function continuous on the union of the spectra of $N_{1}$ and $N_{2}$. If $K$ and $\epsilon$ are greater than zero, then there is $\delta$ greater than zero such that $\|B\| \leq K$ and $\left\|N_{1} B-B N_{2}\right\| \leq \delta$ imply that $\left\|\psi\left(N_{1}\right) B-B \psi\left(N_{2}\right)\right\| \leq \epsilon$.

Proof. The technique used to prove Corollary 1, plus the observation that $\psi\left(N_{1} \oplus N_{2}\right)=\psi\left(N_{1}\right) \oplus \psi\left(N_{2}\right)$, shows that we may assume that $N_{1}=$ $N_{2}=N$; without loss of generality we assume as before that $\|N\|=1$. We use a three-step proof.

Step one. In case $\psi(N)$ is a monomial in $N$ and $N^{*}$, say $\psi(N)=$ $N^{k} N^{* j}$, we have 


$$
\begin{aligned}
\left\|N^{k} N^{* j} B-B N^{k} N^{* j}\right\| & \leq\left\|N^{k} N^{* j} B-N^{k} B N^{* j}\right\|+\left\|N^{k} B N^{* j}-B N^{k} N^{* j}\right\| \\
& \leq\left\|N^{* j} B-B N^{* j}\right\|+\left\|N^{k} B-B N^{k}\right\|,
\end{aligned}
$$

since $N$ has norm 1. By the relation used at the beginning of the proof of the Theorem,

$$
\begin{aligned}
\left\|N^{k} N^{* j} B-B N^{k} N^{* j}\right\| & \leq j\left\|N^{*}\right\|^{j-1}\left\|N^{*} B-B N^{*}\right\|+k\|N\|^{k-1}\|N B-B N\| \\
& =j\left\|N^{*} B-B N^{*}\right\|+k\|N B-B N\| .
\end{aligned}
$$

Choose $\delta^{\prime}$ so that $\|B\| \leq K$ and $\|N B-B N\| \leq \delta^{\prime}$ imply $\left\|N^{*} B-B N^{*}\right\|$ $\leq \epsilon / 2 j$, and let $\delta=\min \left(\delta^{\prime}, \epsilon / 2 k\right)$. Then if $\|B\| \leq K$ and $\|N B-B N\| \leq \delta$ we have

$$
\left\|N^{k} N^{* j} B-B N^{k} N^{* j}\right\| \leq j \cdot \epsilon / 2 j+k \cdot \epsilon / 2 k=\epsilon .
$$

Step two. In case $\psi(N)$ is a polynomial in $N$ and $N^{*}$, say $\psi(N)=$ $\Sigma_{k+j \leq m} \alpha_{k j} N^{k} N^{* j}$, we have

$$
\|\psi(N) B-B \psi(N)\| \leq \sum_{k+j \leq m}\left|\alpha_{k j}\right|\left\|N^{k} N^{* j} B-B N^{k} N^{* j}\right\| .
$$

By Step one, choose $\delta_{k j}$ greater than zero so that $\|B\| \leq K$ and $\|N B-B N\|$ $\leq \delta_{k j}$ imply $\left\|N^{k} N^{* j} B-B N^{k} N^{* j}\right\| \leq \epsilon /\left|\alpha_{k j}\right| m(m+1)$. Let $\delta=\min \left\{\delta_{k j}\right.$ : $k+j \leq m\}$. Then $\delta>0$ and the conditions $\|B\| \leq K$ and $\|N B-B N\| \leq \delta$ imply

$$
\begin{aligned}
\|\psi(N) B-B \psi(N)\| & \leq \sum_{k+j \leq m}\left|\alpha_{k j}\right| \cdot \frac{\epsilon}{\left|\alpha_{k j}\right| m(m+1)} \\
& \leq \sum_{k+j<m} \frac{\epsilon}{m(m+1)} \leq \epsilon .
\end{aligned}
$$

Step three. Now let $\psi$ be any function continuous on the spectrum of $N$. Then there is a polynomial $p(\lambda, \bar{\lambda})$ such that $\sup _{\lambda \in \mathbf{\Lambda}(N)}|p(\lambda, \bar{\lambda})-\psi(\lambda)| \leq$ $\epsilon / 4 K$, where $\Lambda(N)$ is the spectrum of $N$. We then have

$$
\begin{aligned}
\|\psi(N) B-B \psi(N)\| \leq & \left\|\psi(N) B-p\left(N, N^{*}\right) B\right\|+\left\|p\left(N, N^{*}\right) B-B p\left(N, N^{*}\right)\right\| \\
& +\left\|B p\left(N, N^{*}\right)-B \psi(N)\right\| \\
\leq & 2\|B\| \cdot\left\|,(N)-p\left(N, N^{*}\right)\right\|+\left\|p\left(N, N^{*}\right) B-B p\left(N, N^{*}\right)\right\| .
\end{aligned}
$$

Finally, by Step two we can choose $\delta$ greater than zero so that $\|B\| \leq K$ and $\|N B-B N\| \leq \delta$ imply that $\left\|p\left(N, N^{*}\right) B-B p\left(N, N^{*}\right)\right\| \leq \epsilon / 2$. The condition defining the polynomial $p$, plus the spectral theorem, imply that 
$\left\|\psi(N)-p\left(N, N^{*}\right)\right\| \leq \epsilon / 4 K$. Hence for $\|B\| \leq K$ and $\|N B-B N\| \leq \delta$ we have

$$
\left\|\psi^{\prime}(N) B-B \psi(N)\right\| \leq 2 K \cdot \epsilon / 4 K+\epsilon / 2=\epsilon .
$$

The proof is complete.

\section{REFERENCES}

1. J. J. Bastian and K. J. Harrison, Subnormal weighted shifts and asymptotic properties of normal operators, 1973 (preprint).

2. S. K. Berberian, Note on a theorem of Fuglede and Putnam, Proc. Amer. Math. Soc. 10 (1959), 175-182. MR 21 \#6548.

3. P. R. Halmos, What does the spectral theorem say? Amer. Math. Monthly 70 (1963), 241-247. MR 27 \#595.

4. B. E. Johnson and J. P. Williams, The range of a normal derivation, Pacific J. Math. (to appear).

5. Marvin Rosenblum, On a theorem of Fuglede and Putnam, J. London Math. Soc. 33 (1958), 376-377. MR 20 \#6037. 47401

DEP ARTMENT OF MATHEMATICS, INDIANA UNIVERSITY, BLOOMINGTON, INDIANA

Current address: Department of Mathematics, Bucknell University, Lewisburg, Pennsylvania 17837 